\title{
REMARKS ON THE INDUCTIVE PRINCIPLE AND RELATED EXISTENCE THEOREMS $\dagger$
}

\section{BY HENRY BLUMBERG}

It is the purpose of the present note to show the validity, in a natural formulation, of the inductive principle and of related existence theorems for the general linear order and for the $n$-fold order. $f$ The simple relations which these theorems bear to the inductive principle and to one another are brought out tersely. An example shows that the inductive principle is not valid for the general $\boldsymbol{N}_{0}$-fold order.

Let $A$ be a given linear order. By an extension of an initial segment $I$ of $A$-analog of neighborhood of a point-we understand a segment $\S$ of $A$ containing elements of both\| $I$ and $\bar{I}$. If $E(\neq A)$ is a subset of $A$, let $I$ be the set of elements of $A$ preceding all the elements of $E$; we refer to $I$ as the initial segment associated with $E$. While $I$ consists exclusively of elements of $\bar{E}$, every extension of it contains elements of $E$. To deny the existence of an initial segment $I$ thus characterized is to affirm that $\bar{E} \equiv A$. In other words, to say that for every initial segment consisting exclusively of elements of $\bar{E}$ there is an extension consisting exclusively of elements of $\bar{E}$ is to say that $\bar{E} \equiv A$. The inductive principle for a general linear order is thus a rewording of the characteristic property of the initial segment

$\dagger$ Presented to the Society, April 7, 1928.

$\ddagger$ The inductive principle appears here in a different light from that of the view represented, for example, by H. Poincaré in Science and Hypothesis. In this connection, see also Khintchine, Das Stetigkeitsaxiom des Linearcontinuums als Inductionsprinzip betrachtet, Fundamenta Mathematicae, vol. 4 (1923), p. 164, and Hildebrandt, The Borel theorem and its generalizations, this Bulletin, vol. 32 (1926), p. 423.

$\S$ A segment of $A$ is a subset of $A$ containing with every pair of its elements all the elements of $A$ between them.

$\|$ We denote the complement $A-E$ of a set $E$ by $\bar{E}$. If $I=0$, where 0 stands for the null initial segment, we regard every non-null initial segment of $A$ as an extension of $I$; if $I=A$, we regard every non-null final segment of $A$ as an extension of $I$. 
associated with a subset of $A$. We call the element property $\alpha$ inductive for $A$ if its validity for all the elements of an initial segment $I$ of $A$ implies its validity for all the elements of some extension of $I . \dagger$ We thus have the following principle.

Inductive Principle for a Linear Order. If $A$ is a linear order, and $\alpha$ an element-property inductive for $A$, every element of $A$ has property $\alpha$.

If $A$ is normally ordered, it is special in that every initial segment has a first element after it; the inductive principle thus takes the familiar form in this case. If $A$ is the linear continuum, it is special in that every initial segment has a last element, or a first following it, and the inductive principle again reduces to known form.

As an application of the inductive principle, we may derive the Borel Covering Theorem for a general linear order. For suppose that $T$ is a given set of segments of $A$ such that every initial segment of $A$ has an extension which is an element of $T$. Let $A_{1}$ be the linear order whose elements are the initial segments of $A$, it being understood that if $I_{1}$ and $I_{2}$ are two elements of $A_{1}$ such that $I_{1} \subset I_{2}$, then $I_{1} \prec I_{2}$. We say that an element of $A_{1}$ is finitely coverable, if it is contained in the sum of a finite number of elements of $T$. Let $J$ be an initial segment of $A_{1}$ all of whose elements are finitely coverable; $I$ the sum of the elements of $J ; X$ an element of $T$ which is an extension of $I$; $e$ an element of $I X$; and $I(e)$ the set of elements of $A$ preceding $e$. Since $e$ belongs to $I, I(e)$ belongs to $J$ and is therefore finitely coverable. Hence $I+X$ is finitely coverable, and therefore every element of $A_{1}$ lying in $I+X$ is finitely coverable. There thus exists for $J$ an extension all of whose elements are finitely coverable. Accordingly, finite coverability is an inductive property for $A_{1}$, and since $A$ belongs to $A_{1}$, it is finitely coverable. We thus have the following theorem.

Borel Covering Theorem for a Linear Order. If $T$ is a set of segments of the linear order $A$ such that every initial segment of $A$ has an extension which is an element of $T$, there exists a finite subset of $T$ having the same property.

$\dagger$ Every element property is regarded as vacuously valid for the elements of the null set. 
The Borel theorem remains valid if we replace $A$ by a segment $S$ of it, provided the given set $T$ contains an extension of every initial segment of $S$, with the understanding that an extension of the initial segment $I$ of $S$ means an extension of $J+I$, where $J$ is the initial segment of $A$ associated with $S$.

We mention lastly, as immediate implication of the Borel theorem, the existence theorem associated with a descending segment property, that is, a property such that if it holds for a segment $S$ of $A$, and $S$ is contained in the sum of a finite number of segments $S_{1}, \cdots, S_{n}$, it holds for at least one $S_{\nu}$.

If $\alpha$ is a descending segment property, and $S$ a segment of $A$ having property $\alpha$, then for some initial segment of $S$ every extension has property $\alpha$.

For otherwise every initial segment of $S$ would have an extension not having property $\alpha$, and therefore, according to the Borel theorem, and contrary to the descending character of $\alpha$, $S$ would be contained in a finite number of segments none of property $\alpha$.

We next consider a general $n$-fold order $A=A_{1} A_{2} \cdots A_{n}$, the $A_{\nu}$ being any linear orders whatsoever, and the set $A$ consisting of all the elements $a=\left(a_{1}, a_{2}, \cdots, a_{n}\right)$, where the $\nu$ th coordinate $a_{\nu}$ is an element of $A_{\nu}$. By an initial rectangle of $A$, we understand an $n$-fold order $I=I_{1} I_{2} \cdots I_{n}$, where $I_{\nu}$ is an initial segment of $A_{\nu}$. An extension of $I$ is the set of elements in an order $X=X_{1} X_{2} \cdots X_{n}$, where $X_{\nu}$ is an extension of $I_{\nu}$. The element property $\alpha$ will be said to be inductive for $A$, if for every initial rectangle with elements all of property $\alpha$, there exists an extension with elements all of property $\alpha$. We now prove the following principle.

IndUCtIVE PRINCIPLE FOR AN $n$-Fold ORder. If $A$ is an $n$-fold order, and $\alpha$ an element property inductive for $A$, every element of $A$ has property $\alpha$.

Proof. Since the initial null rectangle, that is, the rectangle for which the component linear initial segments are all null sets, has (vacuously) all its elements of property $\alpha$, there is an extension of it, that is, an initial rectangle with every compo- 
nent $\neq 0$, such that every element of it has property $\dagger \alpha$. Let $I=I_{1} I_{2} \cdots I_{n}$ be any given initial rectangle such that $I_{\nu}=0$, $(\nu=1, \cdots, n)$, and every element of $I$ has property $\alpha$. For the order $I_{n}$, let $J_{n}$ be the associated order of its initial segments. We shall say that the element $I_{n}^{\prime}$ of $J_{n}$ has property $\beta$ if there is an extension $X^{(n-1)}=X_{1} X_{2} \cdots X_{n-1}$ of $I^{(n-1)}=I_{1} I_{2} \cdots I_{n-1}$ such that every element of $X^{(n-1)} I_{n}^{\prime}=X_{1} X_{2} \cdots X_{n-1} I_{n}^{\prime}$ has property $\alpha$. Suppose that $J_{n}^{\prime}$ is an initial segment of $J_{n}$ every element of which has property $\beta$. Let $I_{n}^{*}$ be the initial segment of $I_{n}$ which is the sum of the elements of $J_{n}^{\prime}$. Since every element of $I^{(n-1)} I_{n}^{*}$ has property $\alpha$, which is inductive by hypothesis, there is an extension $X_{1} \cdots X_{n-1} X_{n}=X^{(n-1)} X_{n}$ of this rectangle having only elements of property $\alpha$. Let $e$ be an element common to $X_{n}$ and $I_{n}^{*}$. The element $e$ belongs to an element of $J_{n}^{\prime}$, therefore, if $I(e)$ represents the set of elements of $I_{n}$ preceding $e, I(e)$ is a subset of an element of $J_{n}^{\prime}$. Therefore, on account of property $\beta$, there is an extension $X^{\prime(n-1)}=X_{1}^{\prime} X_{2}^{\prime} \cdots$ $X_{n-1}^{\prime}$ of $I^{(n-1)}$ such that every element of $X^{\prime(n-1)} I(e)$ has property $\alpha$. If $X^{\prime \prime(n-1)}$ is an extension of $I^{(n-1)}$ common to $X^{(n-1)}$ and $X^{\prime(n-1)}$, every element of $X^{\prime \prime(n-1)} I_{n}^{\prime}$, where $I_{n}^{\prime}=I(e)+X_{n}$, has property $\alpha$. Every element of $J_{n}^{\prime}$ contained in $I_{n}^{\prime}$ thus has property $\beta$. Property $\beta$ is therefore inductive for $J_{n}$, and is thus valid for all of its elements, in particular for $I_{n}$. Consequently, there is an extension $Y^{(n-1)}=Y_{1} Y_{2} \cdots Y_{n-1}$ of $I^{(n-1)}$ such that every element of $Y^{(n-1)} I_{n}$ has property $\alpha$. Let us now say, holding $I_{n}$ fixed, that an element $e^{(n-1)}=e_{1} e_{2} \cdots e_{n-1}$ of $A^{(n-1)}$ $=A_{1} A_{2} \cdots A_{n-1}$ has property $\alpha^{\prime}$ if $e_{1} e_{2} \cdots e_{n-1} e_{n}$ has property $\alpha$ for every element $e_{n}$ of $I_{n}$. We have shown that if $I^{(n-1)}$ is such that every element of $I^{(n-1)} I_{n}$ has property $\alpha$, there is an extension $Y^{(n-1)}$ of $I^{(n-1)}$ such that every element of $Y^{(n-1)} I_{n}$ has

$\dagger$ It is to be understood that the meaning of vacuous validity is not to be so interpreted as to permit us to reason that if there is at least one component $I_{\nu}$ of the initial rectangle $I=I_{1} I_{2} \cdots I_{n}$ which is the null set, then $\alpha$ is (vacuously) valid for the elements of $I$, and therefore an extension of $I$ exists with all of its elements of property $\alpha$. But while such reasoning is disallowed, we are to understand that it is permissible to conclude that such an extension exists in case it is already known that $\alpha$ is valid for every element of $I^{\prime}$, where $I^{\prime}$ is an initial rectangle with components identical with those of $I$-now assumed all $\neq 0$ except for $I_{\nu}$-except that the $\nu$ th component of $I^{\prime}$ is not the null set. The present remark relates also to the example given below of an $\aleph_{0}$-fold order for which the inductive principle is not valid. 
property $\alpha$. In other words, property $\alpha^{\prime}$ is inductive for the $(n-1)$-fold order $A^{(n-1)}$. Assuming the inductive principle for every $(n-1)$-fold order, we conclude that every element of $A^{(n-1)} I_{n}$ has property $\alpha$. Similarly we conclude that every element of $A^{(n-1)} A_{n}=A$ has property $\alpha$, that is, that $\alpha$ is inductive for $A$.

That the inductive principle need not hold for an $\boldsymbol{N}_{0}$-fold order is seen from the following example. Let $A=A_{1} A_{2} A_{3} \ldots$ be the $\boldsymbol{N}_{0}$-fold order in which every $A_{\nu}$ is the order of the positive integers in ascending magnitude. We shall say that $e=\left(e_{1}, e_{2}, \cdots\right)$ of $A$ has property $\alpha$ if $\sum_{1}^{\infty} 1 / e_{\nu}$ is divergent. Suppose that $I=I_{1} I_{2} \ldots$ is an initial segment of $A$ for all the elements of which $\alpha$ is valid. Let $\mu_{\nu}$ be the last element of $I_{\nu}$, in case there is such a last element. Take as extension of $I$ the $N_{0}$-fold order $X=X_{1} X_{2} \cdots$, where $X_{\nu}$ consists of the pair of elements $\mu_{\nu}, \mu_{\nu}+1$ when $\mu_{\nu}$ exists, and is an arbitrarily chosen extension, that is, an arbitrary non-null final segment of $I_{\nu}$, when $\mu_{\nu}$ does not exist. Since $\sum^{\prime} 1 / \mu_{\nu}$, where the summation $\sum^{\prime}$ applies to the $\nu$ for which $\mu_{\nu}$ exists, is divergent, it follows that $\sum^{\prime} 1 /\left(\mu_{\nu}+1\right)$ is divergent. Therefore $\sum_{1}^{\infty} 1 / e_{\nu}$ is divergent for every element of $X$, so that for every initial rectangle whose elements have property $\alpha$, there is an extension whose elements have property $\alpha$. Therefore $\alpha$ is inductive without being valid for every element of $A$.

This example fortifies the idea that while the passage from a set to its complement is localizable in the case of a linear order, and in a certain distinct sense also in the case of the $n$-fold order, it is not so in the case of the $\boldsymbol{N}_{0}$-fold order.

The Borel theorem and the theorem on the descending segment property are extensible to the $n$-fold order without novelty of method.

In the case of a linear order, we may, of course, substitute for the definitional property of an extension $X$ of an initial segment $I$ the property that $X$ is a non-null initial segment of $A-I$. But if we define extension for an $n$-fold order on the basis of this modification, the inductive principle need obviously no longer hold for an $n$-fold order, with $n \geqq 2$, as we see from the following example: $A=A_{1} A_{2}, A_{1}$ and $A_{2}$ both consisting of the elements $e_{1}$ and $e_{2}$ with $e_{1}<e_{2} ;$ property $\alpha$ is to be valid for $\left(e_{1}, e_{1}\right)$ and $\left(e_{2}, e_{2}\right)$ but not for $\left(e_{1}, e_{2}\right)$ and $\left(e_{2}, e_{1}\right)$.

The Ohio State University 